\title{
Lamps, cubes, balls and walls: Zeno problems and solutions
}

\author{
Jeanne Peijnenburg · David Atkinson
}

Published online: 27 March 2009

(C) The Author(s) 2009. This article is published with open access at Springerlink.com

\begin{abstract}
Various arguments have been put forward to show that Zeno-like paradoxes are still with us. A particularly interesting one involves a cube composed of colored slabs that geometrically decrease in thickness. We first point out that this argument has already been nullified by Paul Benacerraf. Then we show that nevertheless a further problem remains, one that withstands Benacerraf's critique. We explain that the new problem is isomorphic to two other Zeno-like predicaments: a problem described by Alper and Bridger in 1998 and a modified version of the problem that Benardete introduced in 1964. Finally, we present a solution to the three isomorphic problems.
\end{abstract}

Keywords Zeno problems · Benardete paradox

Our understanding of the conceptual problems raised by Zeno's paradoxes was significantly deepened by mathematical achievements in the nineteenth century (cf. Grünbaum 1967; Salmon 1970). Yet new Zeno-like problems continue to crop up. A particularly interesting one was formulated by Miloš Arsenijevic in 1989, and it has remained unsolved to the present day. In this paper we shall do the following:

(1) We first argue that the problem posed by Arsenijevic can be resolved. For it is equivalent to a puzzle posed by James Thomson in 1954-1955, which has been solved by Paul Benacerraf in his celebrated paper of 1962.

\footnotetext{
J. Peijnenburg · D. Atkinson $(\bowtie)$

Faculty of Philosophy, University of Groningen, Oude Boteringestraat 52,

9712 GL Groningen, The Netherlands

e-mail: d.atkinson@rug.nl

J. Peijnenburg

e-mail: jeanne.peijnenburg@rug.nl
} 
(2) Next we will show that there is another problem, not noticed by Arsenijevic, that is immune to Benacerraf's treatment.

(3) We then demonstrate that the new problem is isomorphic to a Zeno-like paradox which has been described by Alper and Bridger (1998).

(4) We argue that the new problem is also isomorphic to a modified version of a Zeno-like problem described by Benardete (1964).

(5) Finally we explain that the three problems (the new problem introduced in 2 , the Alper and Bridger paradox described in 3, and the modified Benardete paradox of 4) can all be solved in the same way. The best approach to the original Benardete paradox, however, is still to be found in Yablo (2000).

(1) Consider a cube built up from slabs according to the following rules. Each slab is $1 \mathrm{~m}$ wide and $1 \mathrm{~m}$ deep, but they are of different heights. The bottom slab is $1 / 2 \mathrm{~m}$ in height and it is colored red. The next slab, laid down on top of the red one, is only $1 / 4 \mathrm{~m}$ high and it is green. The following slab, on top of the green one, is $1 / 8 \mathrm{~m}$ high, and is red again. The construction continues ad infinitum with alternating colors, the heights of successive slabs decreasing in the same geometric fashion. Looking down on the completed cube from above, would one see red or green?

This problem was first described by Miloš Arsenijevic and it is as yet unresolved (Arsenijevic 1989). However, we believe that the cube as described by Arsenijevic is accurately paralleled by the so-called Thomson lamp (Thomson 1954-1955). Aiming to show that we are still in a Zeno-like grip, James Thomson had presented us with a lamp that is turned on at time 0 , off at $1 / 2 \mathrm{~min}$, on at $3 / 4 \mathrm{~min}$, and so on, ad infinitum. Thomson then asks himself: 'Is the lamp on or off 1 min after the start?' The fact that we seem unable to answer that question was regarded by him as an indication that modern versions of the old Achilles are still with us today. However, Paul Benacerraf famously pointed out the flaw lurking in Thomson's question: it is the incorrect supposition that the rules as laid out by Thomson imply that the lamp will be either on or off after $1 \mathrm{~min}$ (Benacerraf 1962). As Benacerraf explains, these rules only tell us that every time the lamp is turned on (off) before $1 \mathrm{~min}$, it is turned off (on) shortly thereafter. Nothing at all is implied concerning the state of the lamp at the 1 min mark. The lamp might be on or it might be off: either possibility is compatible with the rules of Thomson's game-something that Thomson himself roundly admitted in his 'Comments on Professor Benacerraf's Paper':

“Benacerraf's excellent article puts it beyond doubt that much of [my paper] is mistaken”. (Thomson 1970, p. 130).

There is a complete parallel between Arsenijevic's question 'Is the cube red or green after completion?' and Thomson's question 'Is the lamp on or off 1 min after the start?' Just as the lamp's being on or off at the 1 min mark is compatible with the rules of the Thomson game, so the completed cube's being red or green is reconcilable with the rules set by Arsenijevic. Arsenijevic's cube and Thomson's lamp are in fact isomorphic, which becomes clear when we look at the assumptions that Thomson and Arsenijevic make. Thomson explicitly assumes that the lamp has to be either on or off 1 min after the start: 
"[The lamp] cannot be on, because I never turn it on without turning it off. It cannot be off, because I did in the first place turn it on, and thereafter I never turned it off without at once turning it on. But the lamp must be either on or off. This is a contradiction." (Thomson 1954-1955, p. 95; italics added).

In exactly the same manner, Arsenijevic assumes that the cube has to be red or green after completion:

"It may be said that we don't know what the case may be, but, it seems, it is certain that in any case this must be [red] or [green]" (Arsenijevic 1989, p. 37; italics added).

We may formalize Thomson's description of his lamp as follows:

(A) Every time that the lamp is turned on before the 1 min mark, it is then turned off before the 1 min mark.

(B) Every time that the lamp is turned off before the 1 min mark, it is then turned on before the 1 min mark.

(C) The lamp is either on or off at the 1 min mark.

The parallel formalization of Arsenijevic's cube is:

$\left(\mathrm{A}^{\prime}\right)$ Every time that a red slab is laid down, a green slab is then laid on top of it. $\left(B^{\prime}\right)$ Every time that a green slab is laid down, a red slab is then laid on top of it.

$\left(C^{\prime}\right)$ The completed cube is either red or green.

The similarity between the Thomson lamp and Arsenijevic's cube is further evidenced if we put the first (red) slab in place at time 0 , the second slab at $1 / 2 \mathrm{~min}$, the third one at $3 / 4 \mathrm{~min}$, and so on. If we observe the cube from above during its construction, the color will fluctuate in exactly the same way as does the Thomson lamp. After $1 \mathrm{~min}$ the construction will be finished. And just as the state of the Thomson lamp at the $1 \mathrm{~min}$ mark is undetermined, so the color of the cube after $1 \mathrm{~min}$ is not determined either. The fact that the lamp remains the same whereas the cube only gradually takes shape is not relevant here. What does matter is that in both examples we encounter oscillating, nonconvergent sequences of states. In the one case there is an infinite oscillation between the lamp's being on or off, in the other between the cube's looking red or green. In fact, if we were to redesign the lamp so that it switches from red to green rather than from on to off, the color history would be the identical: in either case we would observe a nonconvergent oscillation between red and green.

We conclude that the question Arsenijevic poses concerning his cube is as indeterminate as is the question Thomson posed of his lamp. Moreover, both Arsenijevic's cube and Thomson's lamp involve systems that are logically (although of course not physically) possible. For there is no logical impediment to $\mathrm{A}, \mathrm{B}$ and $\mathrm{C}$ being simultaneously true, and the same goes for the triplet $\mathrm{A}^{\prime}, \mathrm{B}^{\prime}$ and $\mathrm{C}^{\prime}$. Consequently, neither of the two systems constitutes a paradox.

(2) The idea of cubes being built up from slabs according to Zenonian rules gives however rise to another problem, not noticed by Arsenijevic. This problem cuts deeper, and is much more disconcerting than the problem that he describes, for the new problem is immune to Benacerraf's treatment. 
A good way to illustrate the new difficulty is on the basis of a cube with slabs that are all of one color. This helps us to appreciate the essential problem and to distinguish it from the configuration described by Arsenijevic. The monochromaticity is however not essential, as we will show later: the problem can equally well be explained in terms of bicolored slabs.

So let us suppose that all the slabs have the same color-let them all be blue, for example. All the slabs are now identical in every respect, except for their thicknesses: as before, these thicknesses will decrease in the familiar Zenonian way. Now imagine that we have completed our unicolored cube. Like the bicolored cube described by Arsenijevic, our unicolored cube has a total height of $1 \mathrm{~m}$. Looking down on this unicolored cube from a height of $2 \mathrm{~m}$ above the ground, we would clearly see blue-or would we? Which of the infinitely many blue slabs would one in fact see? One would not see a slab at the limiting height, $1 \mathrm{~m}$ from the ground, for there is no slab at this height. Apparently one would have to see a blue slab immediately below the limit point, slightly less than $1 \mathrm{~m}$ from the ground. But which blue slab under the limit point could one see? Any given slab under the limit point is covered by another slab, so no slab at all can in fact be seen! Thus it seems that we would not see anything. But this is surely baffling, given that we simply started by piling up blue slabs on top of one another, and nothing else.

The problem goes deeper. If nothing can be seen of the cube, it must be that no light is reflected from it. But if no light is reflected from it, then the cube must be perfectly absorbing. A black cube. But this will not do either, as can quickly be seen by parallel reasoning. For where would light in fact be absorbed? No light can be absorbed at a height $1 \mathrm{~m}$ above the ground, for there is no slab there to absorb it. If it is to be absorbed, that must occur at one of the slabs below the $1 \mathrm{~m}$ mark; but that cannot happen either, since any slab is covered by another slab, which should have absorbed the light before it could reach the slab in question.

The apparent conclusion is that our pile of blue slabs can neither reflect nor absorb light. Thus it can neither appear blue nor black, nor any other color for that matter. And here the vital difference with the cube as described by Arsenijevic becomes clear. As we have seen, Arsenijevic specified that the cube is red or is green after completion; it is just that the rules do not determine which it is. In the same vein, Thomson assumed that his lamp is on or is off $1 \mathrm{~min}$ after the start; it is just that the rules do not determine which is the true state. In describing our blue cube, however, we have not made the assumption that after completion the cube must be in one of two mutually exclusive states. Hence the question of indeterminacy does not arise. In other words, while Arsenijevic's cube is isomorphic to Thomson's lamp, our cube is not.

We saw that both Arsenijevic's cube and Thomson's lamp constitute systems that are logically possible, in the sense that $\mathrm{A}, \mathrm{B}$ and $\mathrm{C}$ (or $\mathrm{A}^{\prime}, \mathrm{B}^{\prime}$ and $\mathrm{C}^{\prime}$ ) can be true at the same time. Our cube, on the other hand, seems to involve a logical impossibility. For it looks as though the following three propositions cannot all be simultaneously true:

(a) The cube is built from blue slabs that are of geometrically decreasing thicknesses, as described above. 
(b) Every time light impinges on a slab, it is either reflected or absorbed.

(c) Light can be neither reflected nor absorbed by the cube.

Informally, with $\mathrm{b}$ and $\mathrm{c}$ as background assumptions, a cube as described in a apparently cannot exist. ${ }^{1}$

It is clear that the new problem is immune to the treatment of Benacerraf. Benacerraf's analysis shows us that Arsenijevic's question 'Do we see red or green?' is misleading. However, we have shown that the essential problem is not that we cannot see red or green (or that we cannot see blue, in the case of the unicolored cube), it is rather that seeing anything or seeing nothing are alike impossible! On this matter Benacerraf remains silent. His analysis relates to a configuration in which either of two possibilities that are exhaustive and mutually exclusive must be realized (off or on, red or green), but in which there is indeterminacy between them. The situation that we have described is much graver, since neither of two exclusive and exhaustive possibilities (absorption or reflection) can be realized, so the indeterminacy issue is irrelevant.

A cube as described by a, b and $\mathrm{c}$ is logically impossible, but should we call it a paradox? It looks as though we should. For what could be more paradoxical than that we cannot see anything, or nothing, whereas the only thing that we have been doing was to pile up blue slabs, one after the other?

Earlier we remarked that the monochromaticity is not essential to this paradoxical cube, since the problem that we sketched could also be presented in terms of the multicolored cube. This will now be clear. For a bicolored cube, built up from red and green slabs in the familiar way, would be logically impossible too, and essentially for the same reasons. Moreover, a description of such a cube in terms of $\mathrm{a}, \mathrm{b}$ and $\mathrm{c}$ would similarly lead to a paradoxical conclusion: although we have been doing nothing but piling up red and green slabs alternately, we would neither be able to see anything nor to see nothing. ${ }^{2}$

(3) Our second cube, described by a, b and c, bears a striking resemblance to a Zeno-like system described by Alper and Bridger (1998, p. 366). Imagine an infinite number of identical stationary balls, placed in a straight line at the points $1,1 / 2,1 / 4$, $\ldots{ }^{1 / 2}{ }^{\mathrm{n}} \ldots$ etc. (in due course, the balls will have to be thought of as point masses, but we will nevertheless continue to call them 'balls'). So ball $\mathrm{B}_{1}$ is at point 1 , ball $\mathrm{B}_{2}$ is at $1 / 2, B_{3}$ at $1 / 4$, and so on, as in Fig. 1 . Here the symbol ' $x$ ' indicates the point 0 , which is the point of accumulation of the locations of all the stationary balls. ${ }^{3}$ Now imagine another ball, B, identical to the others. B moves with a constant speed along the same line, starting from the point -1 and approaching the point 0 (as in Fig. 2).

\footnotetext{
1 Since proposition $\mathrm{b}$ entails that light can only be reflected or absorbed by the slabs, and proposition a entails that the cube is nothing but the set of all the slabs, it follows that the cube, too, can only reflect or absorb light. No tertium quid is left open. A system in which such a third possibility were allowed would be a completely different one. See footnote 7 for further details.

${ }^{2}$ Of course, in the case of a bicolored cube, proposition a would read: 'The cube is built from red and green slabs that are of geometrically decreasing thicknesses, as described above.'

3 As in the paper by Alper and Bridger, the locations accumulate at 0, whereas the slabs' positions accumulated at a distance of $1 \mathrm{~m}$ above the ground. This difference is of course irrelevant for the argument.
} 


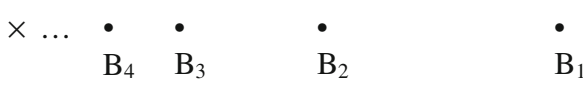

Fig. 1 Infinite number of balls at points $1,1 / 2,1 / 4, \ldots 1 / 2^{\mathrm{n}} \ldots$

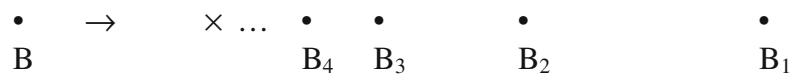

Fig. 2 Ball B moving towards the limit point

What will happen? If B were to collide with one of the stationary balls, and if there were no frictional loss, then B would come to rest. All the energy of B would then be imparted to the other ball. But with which stationary ball would B collide? There is no such ball, for every stationary ball $B_{n}$ has a neighbor $B_{n+1}$ closer to the point of accumulation, with which $B$ should first have collided. This neighbor ball would then have brought B to rest, thereby preventing B's collision with $B_{n}$. But if there is no ball with which B can collide, then B must move on up to point 1 at the right of the line. This is a contradiction.

This system of balls sketched by Alper and Bridger is in fact completely isomorphic to our second cube. The moving ball takes the place of infalling light, and the stationary balls stand in for the slabs, so the Alper and Bridger scenario can be described as:

$\left(a^{\prime}\right)$ Stationary balls are placed at geometrically decreasing distances from point 0 , as described above.

$\left(b^{\prime}\right)$ Every time a moving ball collides with a stationary ball, it comes to rest.

$\left(c^{\prime}\right)$ The moving ball cannot come to rest.

Because the moving ball $\mathrm{B}$ cannot stop at the accumulation point, for there is no ball there with which it could collide, and because B cannot proceed any further, for there are balls in its way, Alper and Bridger draw the conclusion that B must simply cease to exist. This conclusion may well seem far-fetched. How could the laws of mechanics, decribing frictionless collisions between identical balls, lead to the conclusion that a massive ball simply disappears?

In Sect. 5, we will see that there are better ways out of the quandary than the one proposed by Alper and Bridger. But first, in Sect. 4, we will compare our cube with another Zeno-like conundrum, namely the Benardete paradox (Benardete 1964).

(4) In the Benardete scenario, a mortal intends to walk from 0 to 1 . An infinite hierarchy of gods waits in readiness: the $n$th god has the intention to create an impenetrable wall at a point $1 / 2^{\mathrm{n}}$ of the total distance from 0 to 1 , if and only if the mortal reaches that point. So if the mortal were to reach point 1, god-1 would put up wall-1, just in time to prevent him from actually arriving at 1 . If the mortal were to reach $1 / 2$, god-2 would put up wall- 2 , if he were to reach $1 / 4$, god-3 would produce wall-3, and so on. It seems that our man will not be able to proceed beyond point 0 without being blocked by a wall. On the other hand, however, he should be able to walk all the way to 1 , for there can be no walls for him to bump into. After all, no 
god-n can create her wall, since her sister, god- $(n+1)$, should preemptively have created hers.

At first sight, the Benardete system seems very similar to our cube described by $\mathrm{a}, \mathrm{b}$ and $\mathrm{c}$. The parallel can be given as follows:

$\left(a^{\prime \prime}\right)$ The walls are intended to be placed at geometrically decreasing distances from point 0 , as described above.

$\left(b^{\prime \prime}\right)$ If the mortal were to bump into a wall, his progress would be blocked.

$\left(c^{\prime \prime}\right)$ He cannot be blocked by the set of walls.

There is however an important difference between our second cube, as determined by $a, b$ and $c$, and the system given by $a^{\prime \prime}, b^{\prime \prime}$ and $c^{\prime \prime}$. In the latter system, the mortal as well as the gods are merely intending to do something: the mortal intends to walk and each god intends to place her wall. In this sense, the statements $\mathrm{a}^{\prime \prime}, \mathrm{b}^{\prime \prime}$ and $\mathrm{c}^{\prime \prime}$ are describing a battle of intentions rather than a 'battle' amongst slabs trying to reflect and/or absorb light. Another way of phrasing this is by saying that the Benardete scenario is in the conditional mood, whereas the description of our second cube is indicative; the slabs are after all already in place before light falls on the cube. ${ }^{4}$ In order to engender a real isomorphism between our second cube and the Benardete configuration, we have to modify the latter to make it indicative. This is easy enough. All we have to do is to postulate that all the gods create their walls before the mortal begins his walk. The battle is now not in the intentional sphere, but rather in a combination of the geometrical structure at hand (an open set of walls or slabs or stationary balls) and a set of rules concerning the possible progress (of a mortal or of light or of a moving ball):

$\left(a^{\prime \prime \prime}\right)$ Stationary walls are placed at geometrically decreasing distances from point 0 , as described above.

$\left(b^{\prime \prime \prime}\right)$ Every time the mortal bumps into a wall, his progress is blocked.

$\left(\mathrm{c}^{\prime \prime \prime}\right)$ The mortal cannot be blocked by the set of walls.

The system described by $\mathrm{a}^{\prime \prime \prime}, \mathrm{b}^{\prime \prime \prime}$ and $\mathrm{c}^{\prime \prime \prime}$ is isomorphic to the second cube (described by a, b and c) and thus also to the Alper and Bridger scenario (described by $\mathrm{a}^{\prime}, \mathrm{b}^{\prime}$ and $\mathrm{c}^{\prime}$ ).

(5) The original Benardete paradox, described by $a^{\prime \prime}, b^{\prime \prime}$ and $c^{\prime \prime}$, has been astutely analyzed by Yablo (2000). He points out that the difficulty lies in the infinite concatenation of the gods' intentions. For the gods have tied themselves into a logical knot that even they cannot untie. Notwithstanding their divine powers, they are logically unable to carry out their professed plans, since the set consisting of $\mathrm{a}^{\prime \prime}$, $\mathrm{b}^{\prime \prime}$ and $\mathrm{c}^{\prime \prime}$ is simply inconsistent. This has been further deepened by the analysis of Shackel (2005).

However, things are different if we turn to the three other systems that we have discussed: the cube described by $\mathrm{a}, \mathrm{b}$ and $\mathrm{c}$, the Alper and Bridger system given by $\mathrm{a}^{\prime}, \mathrm{b}^{\prime}$ and $\mathrm{c}^{\prime}$, and the modified (i.e. indicative) Bernadete paradox, spelled out by $\mathrm{a}^{\prime \prime \prime}$,

\footnotetext{
${ }^{4}$ Shackel also acknowledges the distinction between what we called the conditional and the indicative mood, but he refers to it in an aside, and does not elaborate the consequences (Shackel 2005, p. 403).
} 
$\mathrm{b}^{\prime \prime \prime}$ and $\mathrm{c}^{\prime \prime \prime}$. These three systems, which are all isomorphic, can be resolved in a manner other than by simply saying that they are internally inconsistent. The fact that this alternate solution does not work for the original Benardete scenario, described by $\mathrm{a}^{\prime \prime}, \mathrm{b}^{\prime \prime}$ and $\mathrm{c}^{\prime \prime}$, is sufficient reason for claiming that there is an essential difference between the original Benardete system on the one hand and the three isomorphic systems on the other.

What is this alternate solution that only works for our three systems? We will first explain it on the basis of the Alper and Bridger scenario, before applying it to the second cube and the indicative Benardete system.

As we have seen, Alper and Bridger 'solve' their paradox by saying that the moving ball B suddenly ceases to exist. We already intimated that we deem this solution to be contrived. It is true that no ball, or any other entity for that matter, ever satisfies an inconsistent system-in that sense ball B must indeed be nonexistent. But as Alper and Bridger would have it, B is not just nonexistent; rather it first exists and then miraculously disappears in a pall of metaphysical smoke. The extravagance of this view is further illustrated if we combine it, as Alper and Bridger do, with the generally accepted principle that the laws of mechanics are time-symmetric. According to this principle, if we take a natural process and consider it in reversed time (as in a film run backward), then the same natural laws should apply. On time reversing Alper and Bridger's solution, we would first see an infinite set of stationary balls at the points $1,1 / 2,1 / 4, \ldots 1 / 2^{\mathrm{n}}, \ldots$ Next we would perceive a new ball suddenly coming into existence at the limit point 0 , and it would then move to the left, towards point -1 . The idea that an entire moving ball (or point mass) springs into existence, thereby providing us with nothing less than a creatio ex nihilo, is so staggering that we may well be forgiven for doubting the cogency of Alper and Bridger's solution.

In our view, Alper and Bridger's claim that ball B disappears is an artefact of an inadequate definition of what a collision is. From their writings it is clear that Alper and Bridger interpret the term 'collision' as 'making contact': when two bodies $\mathrm{X}$ and $\mathrm{Y}$ collide, then $\mathrm{X}$ and $\mathrm{Y}$ come in contact with one another, in the sense that at least one point of $\mathrm{X}$ occupies the same location in space as does one point of $\mathrm{Y}$. Although this definition of 'collision' as 'spatial coincidence' may perhaps accord with common sense, it can scarcely withstand the scrutiny of physicists. Physicists describe 'collision' in terms of a force field in the limit that the range of the force tends to zero. ${ }^{5}$ In their terminology, two bodies collide if and only if the distance between them is zero. If the bodies in question are 'normal' bodies such as balls, or finite sets of balls, then the difference between the first and the second definition is not important. But if one of the bodies constitutes an open set, as is the case with our infinite set of stationary balls placed at $1,1 / 2,1 / 4, \ldots 1 / 2^{\mathrm{n}}, \ldots$, then the difference is essential, for then the two concepts of 'collision' are by no means equivalent. If the moving ball B arrives at the point 0 , then there is no collision between B and any other ball under the first interpretation of 'collision', for there is no ball at 0 with which B can come into contact. But under the definition of 'collision' as 'zero

\footnotetext{
${ }^{5}$ Cf. Leonard Angel, who notes that collision—he calls it 'impact'—need not imply making contact in the spatial sense (Angel 2001, p. 357).
} 
distance', B does indeed collide with the set of stationary balls at the point 0 . Rather than trying to progress beyond the point of accumulation (as suggested by the terms of the paradox) and rather than being annihilated (as Alper and Bridger would have it), B simply bounces back. This can be explicated as follows.

Imagine a repulsive force, $\mathrm{F}$, that is reciprocally exerted between two bodies $\mathrm{X}$ and Y. For simplicity of visualization, we postulate the existence of a threshold, $t$, such that $\mathrm{F}$ is only effective if the distance between $\mathrm{X}$ and $\mathrm{Y}$ is smaller than $\mathrm{t}$. For example, if $t=1 \mathrm{~mm}$., then $\mathrm{X}$ and $\mathrm{Y}$ repel one another if and only if they are not further apart than $1 \mathrm{~mm}$. The force is said to be of finite range.

Now substitute for $\mathrm{X}$ the moving ball $\mathrm{B}$, and for $\mathrm{Y}$ the open set of identical stationary balls. If all the balls of the set are further from B than $1 \mathrm{~mm}$., there is no repulsive force operating, but if some balls are within the range $1 \mathrm{~mm}$., then $\mathrm{B}$ is repelled by these balls. Since each stationary ball $B_{n}$ within the range $1 \mathrm{~mm}$. from $B$ has a ball $B_{n+1}$ between itself and $B$, it is the case that, if there is one ball within the range 1 from $B$, then there is an infinite number of them. And the number remains infinite, even if we take for t not 1 , but for example 0.1 or $0.01 \mathrm{~mm}$. No matter how small the range of the force is, the number of balls within that distance would be infinite if there were any at all within the distance in question. The repulsive forces exerted by all these balls are additive, and if we assume for the sake of argument that each ball within the range contributes the same force $\mathrm{F}$, the total force will be infinite. This situation persists even in the formal limit that $t$ goes to zero. However, B will only be subjected to this force for an infinitesimal duration. Thus the force is impulsive, and as a result B rebounds-that is to say, its velocity is simply reversed.

We conclude that the Alper and Bridger situation does not constitute a paradox if we interpret ' $\mathrm{X}$ collides with $\mathrm{Y}$ at some instant' as 'the distance between $\mathrm{X}$ and $\mathrm{Y}$ becomes zero at some instant'. Under this definition of collision, which is the usual one for physicists, it is not the case that B is obstructed and not obstructed at the same time. So there is no need for exotic 'solutions' like the stipulation that B all at once ceases to exist. On the contrary, what happens to B is what happens to any hard ball that hits a hard ground in a frictionless environment: it bounces back with the same speed as it had when it struck the ground. ${ }^{6}$

A similar story can be told about the indicative Benardete paradox. There the walls are already in place before the mortal starts his walk, and the alleged paradox consists in the fact that the mortal simultaneously is and is not hampered from moving on. He is hampered, because he can never get to point 1 (or even get beyond his starting point 0), since an infinite number of walls will be in his way. At the same time he is not hampered, for there are no walls he can encounter, since in front of every wall-n there is a wall- $(n+1)$ which the mortal should have bumped into first. However, like the term 'colliding with', the term 'bumping into' has two interpretations. The one is the everyday but imprecise notion of 'making contact' and under this interpretation the paradox subsists. The other one is the more precise interpretation of 'having zero distance'. Under this interpretation, the mortal is immediately stopped in his tracks: right at the beginning of his walk, he is halted by

\footnotetext{
${ }^{6}$ These conclusions are confirmed by large finite numerical simulations (D. Atkinson and P.W. Johnson, work in progress).
} 
the massed walls, simply because his nose is separated from them by a zero distance, and whatever repulsive forces one wishes to invoke prevent his further progress.

It will be clear that this solution to the indicative version is powerless in the face of the original Benardete system. For as we have seen, the latter is not in the indicative, but rather in the conditional mood. It does not deal with pukka walls, but only with intentions to create them. Concerning intentions of this sort, physical theories about colliding bodies have nothing to say. Hence the best approach to the original Benardete system still is Yablo's, which makes it clear that the wallbuilders intentions cannot be fulfilled.

However, our solution does work for the second cube. As we have seen, the paradox here is that light impinging on a slab is simultaneously either reflected or absorbed and neither reflected nor absorbed. It is reflected or absorbed, because the stream of photons that make up the light impinge on a pile of slabs. But it is neither reflected nor absorbed because no slab in the pile can actually be irradiated by photons: every slab-n has a neigbor slab- $(n+1)$ upon which the photons should have first impinged. Like 'collide with' and 'bump into', the term 'impinge upon' is however ambiguous. It could mean that the photons make contact with the slabs, or that the distance between the photons and the slabs is zero. The first interpretation may seem commonsensical, but when we are talking about such things as open and bounded sets, the second, scientific interpretation is more apposite. Moreover, while under the first interpretation there is indeed a paradox (or at least an inconsistency), under the second interpretation there is no problem at all: the photons will simply be either reflected or absorbed. ${ }^{7}$

Acknowledgements We thank the Department of Philosophy at the National University of Singapore for providing a congenial ambiance in which we could finish this paper. We also thank our colleagues at the Faculty of Philosophy in Groningen for allowing one of us to absent herself from a number of

\footnotetext{
7 Or transmitted. For as far as the cube is concerned, there is another, very plausible way to remove the inconsistency between $\mathrm{a}, \mathrm{b}$ and $\mathrm{c}$. If we replace $\mathrm{b}$ by:

(b*) Every time light impinges on a slab, it is either reflected, or absorbed or transmitted,

then the paradox disappears. For the unicolored cube, note that a slab will reflect some blue light and absorb some non-blue light, but it will transmit most of the (blue and non-blue) light. The extremely thin layers just below the $1 \mathrm{~m}$ mark will allow almost all light to go through unimpeded, while the thicker layers underneath will reflect more blue light and absorb more non-blue light. If we were to look down on a cube described by propositions like $a, b^{*}$, c, we would see blue.

Note that the replacement of $b$ by $b^{*}$ not only removes all inconsistency in the description of the monochromatic cube, but also in that of the bicolored one. Moreover, as in the monochromatic case, we can give a definite answer to the question which color would be observed when the completed cube is illuminated by white light from above. In the case of the monochromatic cube, this color would be blue. But in the case of the bicolored cube, a thin red slab would reflect some red light and absorb some light of other colors, but most light would be transmitted. Similarly for a thin green slab, mutatis mutandis. Again, the extremely thin layers just below the $1 \mathrm{~m}$ mark will allow almost all light through, while the thicker layers underneath will absorb or reflect more light preferentially, depending on the color. Such a stack of slabs indeed approaches more closely the behavior of real slabs of colored material. However, for such a slab there is no logical, nor even a physical problem (apart from the fact that the number of slabs is infinite): some of the red, and some of the green light will find its way back upwards. Since much of the light is absorbed in or transmitted through the slabs, and only a fraction of both red and green light is reflected back up out of the cube, in the end the cube will look gray.
} 
meetings. An earlier version of this paper has been presented to the members of the Formal Epistemology Project in Leuven, Belgium.

Open Access This article is distributed under the terms of the Creative Commons Attribution Noncommercial License which permits any noncommercial use, distribution, and reproduction in any medium, provided the original author(s) and source are credited.

\section{References}

Alper, J. S., \& Bridger, M. (1998). Newtonian supertasks: A critical analysis. Synthese, 114, 335-369. doi:10.1023/A:1005057130067.

Angel, L. (2001). A physical model of Zeno's dichotomy. The British Journal for the Philosophy of Science, 52, 347-358. doi:10.1093/bjps/52.2.347.

Arsenijevic, M. (1989). How many physically distinguished parts can a limited body contain? Analysis, 49, 36-42. doi:10.2307/3328896.

Benacerraf, P. (1962). Tasks, super-tasks, and the modern eleatics. Journal of Philosophy, 59, 765-784. [Reprinted in Salmon, 1970, 103-129].

Benardete, J. (1964). Infinity: An essay in metaphysics. Oxford: Clarendon Press.

Grünbaum, A. (1967). Modern science and Zeno's paradoxes of motion. Middletown, CT: Wesleyan University Press.

Salmon, W. C. (Ed.). (1970). Zeno's paradoxes. Indianapolis, IN: Hackett Publishing Company. Second, revised edition of 2001.

Shackel, N. (2005). The form of the Benardete dichotomy. The British Journal for the Philosophy of Science, 56, 397-417. doi:10.1093/bjps/axi121.

Thomson, J. (1954-1955). Tasks and super-tasks, Analysis, 15, 1-13. [Reprinted in Salmon, 2001, 89102].

Thomson, J. (1970). Comments on Professor Benacerraf's paper. In: W. C. Salmon (Ed.). Zeno's paradoxes, 1970 (pp. 130-138). [Reprinted in Salmon 2001].

Yablo, S. (2000). A reply to new Zeno. Analysis, 60, 148-151. doi:10.1111/1467-8284.00217. 